\title{
Stroboscopic High-Duty-Cycle GHz Time-Resolved Microscope: Toward Hardware Implementation and Commissioning
}

Jiaqi Qiu ${ }^{1}$, Stanislav S. Baturin ${ }^{1}$, Yingjie Li $^{1}$, Chunguang Jing ${ }^{1}$, Alexei Kanareykin ${ }^{1}$, Sergey V. Baryshev $^{1 *}$, Bryan W. Reed ${ }^{2}$, Daniel Masiel ${ }^{2}$, June W. Lau ${ }^{3}$, Yimei Zhu ${ }^{4}$

1. Euclid TechLabs, 365 Remington Blvd., Bolingbrook, IL 60440, USA

2. Integrated Dynamic Electron Solutions, 5653 Stoneridge Dr., Suite 117, Pleasanton, CA 94588, USA

3. Materials Science and Engineering Division, National Institute of Standards and Technology, Gaithersburg, MD 20899, USA

4. Department of Condensed Matter Physics and Materials Science, Brookhaven National Laboratory, Upton, NY 11973, USA

*s.baryshev@euclidtechlabs.com

Introducing a photocathode and a laser beam into the high voltage electron gun of a transmission electron microscope (TEM), enables two distinct modes of operation: (1) ultrafast or stroboscopic pumpprobe time-resolved electron microscopy/diffraction, dubbed as UTEM - reversible processes are recorded, and (2) dynamic or single-shot pump-probe time-resolved electron microscopy/diffraction, dubbed as DTEM - irreversible instantaneous processes are recorded. In UTEM, data are repeatedly collected over extended periods of time, and thermal load from the pump laser must be handled so a process under study stays reversible. Therefore, even though lasers with higher repetition rates are available, UTEM systems typically operate at much less than $0.1 \mathrm{GHz}$, and sometimes even at $\sim 0.1$ $\mathrm{MHz}$, depending on the experiment. In contrast to most laser-driven processes, many processes in nanodevices driven electromagnetically can be cycled indefinitely at $\mathrm{GHz}$ frequencies, thus enabling in operando microscopy. This class of problems, characterized by electrical stimulus and lack of an extended cool-down time, is largely distinct from the class of problems typically studied in laser-based UTEM systems. Thus a purely electrical approach to pump/probe electron microscopy complements existing laser-based approaches by reaching much higher repetition rates in the study of processes that can be so driven. High repetition rate has an immediate advantage in terms of the amount of time required to accumulate a measured signal because of the potential for much higher duty cycles and thus much higher time-averaged probe current.

To enable such in operando microscopy, recently a compact $\mathrm{GHz}$ pulsing device based on a series of RF modulators and magnetic quadrupoles that is compatible with JEOL TEMs was proposed [1]. The GHz pulser modulates and chops the incoming dc electron beam and converts it into pico- and sub-picosecond electron pulse trains at $>1 \mathrm{GHz}$ repetition rates (see Fig.1), as well as controllably manipulates the resulting pulses. The temporal pulse length and repetition rate for the $\mathrm{GHz}$ pulser can be continuously tunable over wide ranges. The basic principle of such stroboscopic high duty cycle $\mathrm{GHz}$ TEM is presented in Fig.2. A small part of the RF signal to the pulser is diverted to the sample through a phase-locked delay line, such that the sample and the pulser can be perfectly synchronized through the main clock. Near- in operando examination of fast devices is feasible, since they are electromagnetically driven with external power level identical to realistic operation conditions.

At Euclid's R\&D facility, a microscopy horizontal beam-line was established. A $200 \mathrm{keV}$ electron gun of a JEOL 2000FX TEM was fully reassembled, reprogrammed and recommissioned (Fig.2). The gun delivers electron beam of tens of $\mu \mathrm{A}$ at any energy between 0 and $200 \mathrm{keV}$ as imaged by a phosphor 
screen right after the main vacuum gate valve of the gun (Fig.2). It will serve as a high energy high current source to test and commission the GHz pulser. Downstream of the GHz pulser, a streak camera will be installed to characterize the resulting electron pulse trains as well as their transverse and longitudinal coherence. All components - C0 condenser lens, RF modulators, magnetic quadrupoles, vacuum/radiation shell - are currently in fabrication. Here we demonstrate our first experiments with the generated pulse trains at $1-10 \mathrm{GHz}$ repetition rates and $\sim 10 \mathrm{ps}$ pulse lengths.

[1] J Qiu, G Ha, C Jing, SV Baryshev, BW Reed, JW Lau, Y Zhu, Ultramicroscopy 161 (2016), p. 130. [2] We thank Joe Bricker (QSO) and Shintaro Yazuka (JEOL) for their kind help and advice. This work was supported by DOE BES SBIR program Grant no. DE-SC0013121. Yimei Zhu was supported by the U.S. Department of Energy, Office of Science, Office of Basic Energy Sciences, under Contract no. DESC0012704.

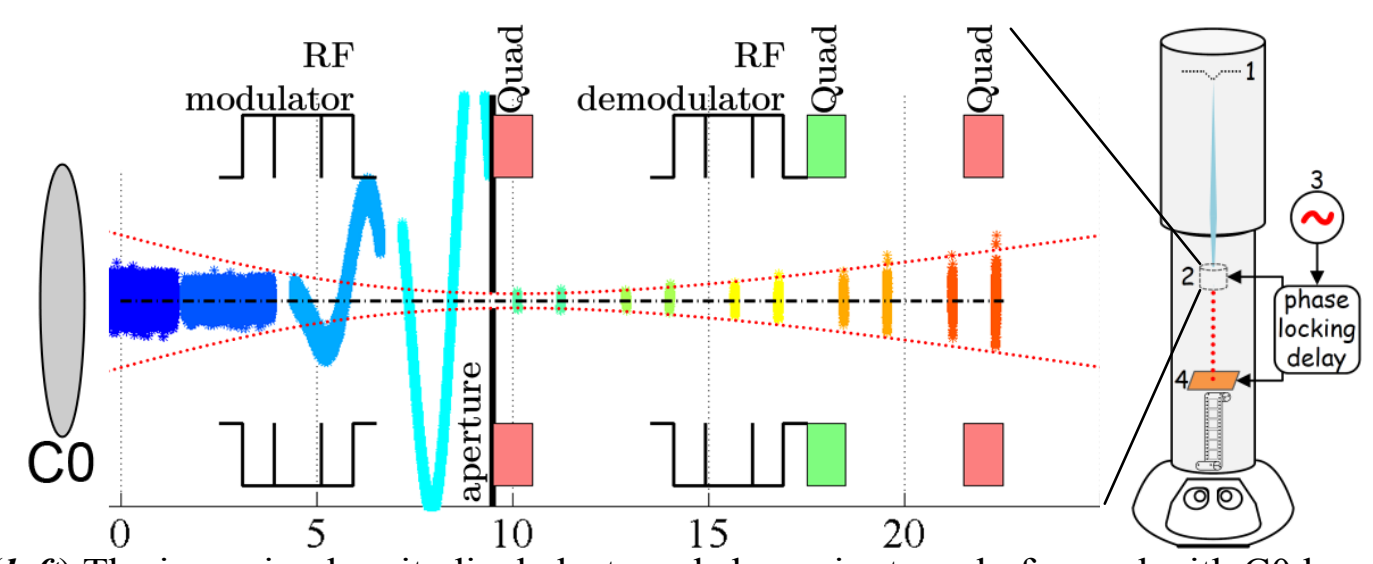

Figure 1. (left) The incoming longitudinal electron dc beam is strongly focused with $\mathrm{C} 0$ lens at the exit of the $200 \mathrm{keV}$ gun and picks up a transverse sine momentum in the first RF modulator. The amplitude of the sinusoid grows as the modulated beam propagates. A collimating aperture is placed on axis to chop the beam and converts it into a pulse train. The finalized design of the GHz pulser has two RF modulators to add/remove transverse momentum and three magnetic quadrupoles to focus and stigmate the beam. Red dotted lines represent beam envelop (not to scale). The central black dash-dotted line is the optical axis. (right) Basic principle of the stroboscopic laser-free high-duty cycle TEM: 1 is the electron dc source; 2 is a dc beam pulsing device; 3 is an RF source; 4 is the sample.
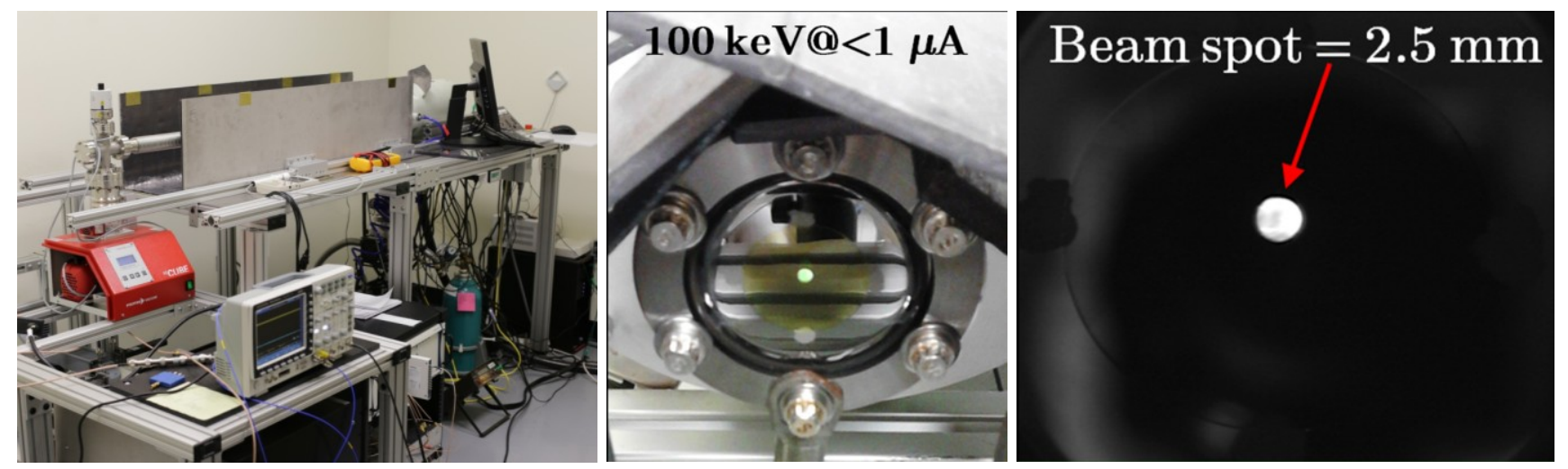

Figure 2. (left) Finalized beam-line with external radiation shielding. (center and right) Beam images on a YAG screen, $7 \mathrm{~cm}$ downstream of the main gate valve of the high voltage gun section. 